III. Religiöse Kontexte und Praxisfelder 


\section{Kontinuität der Nächstenliebe. \\ Die Caritas Steiermark in Zeiten der Pandemie}

Isabelle Jonveaux und Herbert Beiglböck

\section{Einleitung}

Als Hilfs- und Katastrophenhilfe-Organisation ist ,Krise für die Caritas immer auch Teil ihrer Normalität. Hilfe für Menschen in Not kann als Hauptaufgabe der Caritas angesehen werden und bedeutet nichts anderes, als Menschen zu helfen, die sich in einer strukturellen oder persönlichen Krise befinden. Trotz der Erfahrung im Umgang mit Krisen war die Caritas in den Jahren 2020 und 2021 intensiver gefordert als zuvor, denn: Die durch die Pandemie hervorgerufene Krise trifft nicht nur sämtliche Bereiche einer Gesellschaft, sondern beeinflusst eine Hilfsorganisation auch auf ihrer Makro-Ebene. Im Folgenden wird es darum gehen, wie die Caritas der Diözese Graz-Seckau auf die COVID-19-Krise reagiert und ihre Hilfe den Gegebenheiten angepasst hat. Dabei wird besonderes Augenmerk gelegt auf die Spannung zwischen Krise und Normalität, und darauf, wie die Caritas die Kontinuität ihres Dienstes der Nächstenliebe in Zeiten der Krise sicherstellen konnte.

Der vorliegende Beitrag stützt sich auf acht qualitative halb-strukturierte Interviews, die mit LeiterInnen und MitarbeiterInnen verschiedener Einrichtungen der Caritas der Diözese Graz-Seckau im April und Mai 2021 geführt wurden. Diese Beispiele gelten als exemplarisch für die Situation der Caritas während der viel zitierten ,Corona-Pandemie', ohne dabei den Anspruch auf Vollständigkeit zu erheben. In einem ersten Teil wird aufgezeigt, wie die Caritas-Einrichtungen versucht haben und weiterhin versuchen, ihr Werk der Nächstenliebe fortzusetzen. In einem zweiten Teil werden die Herausforderungen untersucht, die sich aus dem Anspruch der Fortsetzung des Auftrags der Nächstenliebe ergeben haben. Abschließend wird gezeigt, wie die Caritas sowohl kurz-, als auch langfristige Systemrelevanz innerhalb einer Krise hat. 


\section{Trotz Krise Caritas bleiben: auf Nähe und Menschen nicht verzichten}

Nach Erwing Goffman sind ,normale Situationen` solche, deren Bedingungen es den sozialen Subjekten erlauben, ihre täglichen Aktivitäten auszuführen, ohne darauf achten zu müssen, was um sie herum geschieht. Dies sind die Situationen, deren Stabilität ein hohes Maß an Vorhersehbarkeit hinsichtlich zukünftiger Ereignisse gewährleistet; sie sind von einer emotionalen Ruhe begleitet, die auf das Fehlen jeglicher Beunruhigung, Bedrohung oder Vorahnung des Unerwarteten zurückzuführen ist (Goffman, zitiert nach Alonso/Bertrand 2011, 2).

Gemäß dieser Definition ist eines klar: Die Situation, in der sich die Caritas und die Gesellschaft als Ganzes seit März 2020 durch die COVID-19-Pandemie befinden, ist keine herkömmliche, keine normale. Die MitarbeiterInnen und Führungskräfte der Caritas mussten nämlich ständig darauf achten, was um sie herum geschah; sie mussten sich an neue Maßnahmen anpassen, während sie gleichzeitig weiterhin auf die üblichen Bedürfnisse ihrer Einrichtungen antworten mussten. Krise meint in diesem Sinne eine ständige Spannung zwischen Normalität und Anomalie. Krisen sind Roux-Dufort zufolge „plötzliche Störungen, an denen mehrere Akteure beteiligt sind, die in einem Klima großer Ungewissheit über den Verlauf der Ereignisse und die Folgen von Entscheidungen sofortige Aufmerksamkeit erfordern" (Roux-Dufort 2003, 16-17). Vor allem in der Anfangsphase der Krise herrschte Unsicherheit, und diese Unsicherheit war auch auf politischer Ebene vorhanden. Der Leiter der Caritas-Einrichtung ,Marienstüberl' in Graz sagte dahingehend: „Die Hauptherausforderung war natürlich die Unsicherheit: Wann kommt das Virus? Wie gefährlich ist es?" Diese Unsicherheit, besonders in der ersten Phase der Pandemie, war in sämtlichen Caritas-Einrichtungen erkennbar. Die Aufgabe der Caritas ist „füreinander da zu sein, an das Gute zu glauben und [zu] helfen, wenn uns jemand braucht" (Caritas 2021). Wie Gabriele Anderssohn, Regionalkoordinatorin für Ennstal und Ausseerland es sagt: „[I]n der Krise ist es der Job der Caritas, da zu sein." Erstes Anliegen der Caritas in der Krise ist also, ihre Aufgaben auch weiter zu erfüllen, soweit das möglich ist. $\mathrm{Zu}$ diesem Zweck hielt die Caritas ihre Einrichtungen, soweit es die offiziellen Maßnahmen der Regierung zuließen, offen.

\subsection{Pflegewohnhäuser: Zwischen Alltag und akuter Krise}

Im Jahr 2019 wohnten 1.165 SeniorInnen in einem der 17 Pflegewohnhäuser der Caritas Steiermark, die sich in den Regionen der ganzen Steiermark 
befinden. Diese Pflegewohnhäuser waren während der gesamten Pandemie geöffnet - selbst dann, als Häuser anderer Organisationen schließen mussten. So weit wie möglich versuchten LeiterInnen und MitarbeiterInnen der Pflegewohnhäuser eine Beibehaltung der Normalität. Anita Freismuth, Leiterin des Pflegewohnhauses in Hitzendorf, fasst es so zusammen: „So viel Normalität wie möglich, so viel Sicherheit wie nötig." In einem Brief an der MitarbeiterInnen schrieb Caritas-Direktor Herbert Beiglböck im September 2020: „Der englische Soziologe Ralf Dahrendorf hat in den 90-iger Jahren einmal darauf hingewiesen, dass Krisensituationen am besten durch gesteigerte Normalität bewältigt werden können. Das klingt sehr einfach, ist aber ebenso anspruchsvoll." Die MitarbeiterInnen in den Caritas-Pflegewohnhäusern legten großen Wert darauf, den Alltag für die BewohnerInnen so normal wie möglich zu gestalten. Unter Einhaltung eines größeren Abstandes zum Sitznachbarn wurde auch weiterhin gemeinsam gegessen - außer in der Zeit, in der es COVID-19-Erkrankungen in der eigenen Einrichtung gab. Gelebte Normalität während der Krise ist aber nur durch Anpassung möglich und wird zu einer, anderen' Normalität. Allerdings braucht es seitens der MitarbeiterInnen mehr Energie, um Normalität aufrecht zu halten. Klaus Sauseng, Leiter der Pflegewohnhäuser in Rottenmann und Frauenberg in der Steiermark, erzählt, wie die MitarbeiterInnen selbst versuchten, den Mangel an Normalität in bestimmten Dimensionen durch die Steigerung anderer Arten von Aktivitäten zu kompensieren:

„Wir als Haus haben ganz stark in die Animation, in den täglichen Ablauf, eingegriffen [...] und mehr Angebote gehabt. [...] - um trotzdem eine gewisse Normalität weiterlaufen zu lassen. Zum Beispiel haben wir uns selbst als Krampus verkleidet, oder als Nikolo. Wir haben diese Feste, die bei uns einen hohen Wert haben, trotzdem immer weiter gefeiert. Wir haben vermehrt Kuchen gebacken, wir haben vermehrt gezeichnet, vermehrt gebastelt. [...] Wir waren aktiver.“

Die pandemiebedingten Sicherheitsmaßnahmen haben einen wesentlichen Teil des Alltagslebens in den Wohnhäusern verändert. Insbesondere hat sich das Besuchsverbot direkt auf das Wohlbefinden der BewohnerInnen ausgewirkt. Klaus Sauseng betont: „Dieses Beschränken der Besuche war für jeden einzelnen Bewohner eine Wegnahme von Gewohnheiten.“ Außerdem wurden verschiedene Aktivitäten oder Veranstaltungen, wie beispielsweise Gottesdienste, abgesagt. Einzelne Veranstaltungen konnten allerdings gelebt werden, wie etwa die Palmweihe oder Muttertag, aber ohne Gäste von außerhalb. „Von einem offenen Haus ist es ein geschlossenes Haus geworden“, sagt dazu die Leiterin des Pflegewohnhauses in 
Hitzendorf. Sobald Besuche wieder möglich waren, wurde ein Besuchsmanagement eingerichtet. An die Stelle der Spontaneität trat eine gewisse Komplexität in das soziale Leben, das die Koordination einer Arbeitskraft erforderte. Akutere Krisen traten in den Pflegewohnhäusern auf, als es COVID-19-positive Fälle gab, sowohl unter den BewohnerInnen als auch den MitarbeiterInnen. Von da an wurde es schwieriger, Normalität aufrechtzuerhalten, da Sicherheit an erster Stelle stand. Im Pflegewohnhaus Sankt Peter in Graz etwa, wo im September 2020 elf BewohnerInnen mit COVID-19 verstarben, begann die Krise mit einer Phase des Chaos.

„Am Anfang der Geschichte, die Chaosphase. Die anstrengendste Phase. Du hast auf einmal viel Arbeit, aber einen großen Ausfall an Personal, sofort. Man kommt von dieser Chaosphase hinaus, wenn das Personal aus der Quarantäne entlassen worden ist und wieder mehr Ordnung reingebracht hat.“ (Klaus Sauseng)

$\mathrm{Zu}$ diesem Zeitpunkt war es nicht mehr möglich, Normalität aufrecht zu erhalten, und das Lebensumfeld der BewohnerInnen wurde erheblich verändert:

„Polstermöbel, Sitzgarnituren, das einmal hinauszuschmeißen, weil es da sein kann, dass es Mikroorganismen gibt, die infektiös sind. Das Loslassen von jahrelang aufgebautem Alltag. Diese Chaosphase war für einige, ich sage nicht nur für BewohnerInnen, sondern auch fürs Personal... Es ist ein Wandel, und dieser Wandel lässt sich definieren über das komplette Demontieren meines gewohnten Pflegewohnhauses. Auf einmal heißt es: Schaffe mir sterile Flächen. Und die Gänge mussten leer sein. Und auf einmal begeben wir uns in ein krankenhausartiges Setting. Die Gänge waren voll mit Sesseln, mit Möbelstücken. Auf einmal hast du den Switch vom gewohnten Umfeld in ein hygienisches, krankenhausartiges Umfeld. Es war zu bemerken, dass viele damit nicht umgehen konnten." (Klaus Sauseng)

Der Rahmen des täglichen Lebens ist mit einer gewissen Sicherheit verbunden und ist umso wichtiger für Menschen, die sich nicht in einer a priori normalen Situation befinden - so etwa ältere Menschen, die nicht mehr zu Hause leben können. Die Beseitigung dieses Rahmens ist dann umso schwieriger und fügt der Krise eine weitere hinzu. 


\subsection{Suppenküche und Lebensmittelausgabe}

Die Suppenküche ,Marienstüberl' in Graz hilft Menschen in akuter Not mit Essen. Im Jahr 2019 etwa wurden dort 55.000 Mittagessen ausgegeben. Andere Suppenküchen, wie etwa die Tafeln vom Roten Kreuz, wurden geschlossen, aber laut Philipp Friesenbichler, einer der beiden Leitungspersonen des Marienstüberls, war „der Gedanke zuzusperren [...] überhaupt nicht da“ und „es wäre schlimm gewesen zu schließen“. Die Bedingungen, unter denen dieser Dienst durchgeführt wurde, insbesondere die Tatsache des gemeinsamen Essens ohne Maske im selben Raum, machten es nichtdestotrotz unmöglich, ihn in der gleichen Form weiterzuführen. Nur Obdachlose durften im Speisesaal essen, das heißt, nicht mehr als zehn Personen. „Im Stüberl hat es einen kleinen Teil der Gäste gegeben - diejenigen, wo wir der Meinung waren, sie haben keine andere Bleibe, sie sind tatsächlich obdachlos, ohne andere Gründe dafür, dass sie stationär essen“, erklärt Philipp Friesenbichler. Für die anderen wurde während den Lockdowns Frühstück und Mittagessen to go in Behältern organisiert und auf dem Parkplatz im Freien ausgeteilt. Die Aufrechterhaltung einer gewissen Normalität war auch eine Säule des Krisenmanagements im Marienstüberl. So etwa betete Schwester Elisabeth Gruber, Leiterin der Einrichtung, auch weiterhin jeden Tag mit den KlientInnen. „Wir beten das Vater Unser, das Gegrüßt seist du Maria, Ehre sei dem Vater und ein kurzes Tischgebet. [...] Wir machen das das ganze Jahr hindurch, wir haben nie aufgehört. Und dann haben wir noch die Kapelle, wo wir auch jeden Tag beten."

Normalität in Krisenzeiten muss tatsächlich neu definiert und rekonstruiert werden; sie ist nicht sofort gegeben. Arbeit ist notwendig, um diese Kontinuität von Normalität zu ermöglichen, und dies erfordert mehr Engagement der MitarbeiterInnen. „Am Anfang war es ein bisschen chaotisch, weil es keine Vorwarnung für die Situation gab. [...] Die ersten drei, vier Tage waren sehr intensiv, weil wir etwas gebraucht haben, was für uns sehr wichtig ist, bei der Arbeit im Stüberl, wir brauchen auch in der Krisensituation einen geregelten Ablauf", so Philipp Friesenbichler. Der geregelte Ablauf ist in Wirklichkeit ein neuer Standard für KlientInnen und MitarbeiterInnen: Er wird den Anforderungen der Krise gerecht und garantiert eine neue Form der Normalität.

Krisenmanagement bedeutet auch, dass die Caritas ihren Horizont erweitern muss, was ihre Klientel anbelangt, denn: Aus einer Krise erwachsen spezifische Bedürfnisse, die erfüllt werden müssen. Um die Schließung der ,Tafeln' des Roten Kreuz zu kompensieren, organisierte die Caritas eine zusätzliche Lebensmittelausgabe in der Herrgottwiesgasse in Graz. In den Regionen, wo es ebenso keine Tafel mehr gab, mussten Lösungen 
gefunden werden. Im Bezirk Liezen beispielsweise wurde am Gründonnerstag 2020 eine Lebensmittelausgabe in drei Orten bis Juni mit der Unterstützung von einem Bus - genannt Carli (Caritas Lieferservice) - ins Leben gerufen. Nach Juni wurden die Lebensmittelausgaben lokal organisiert, in Schladming wurde daraus ein wöchentlicher Sozial-Vital-Markt im Pfarrkindergarten.

\subsection{Kontakt trotz Abstand: Kontinuität in der Beratung und Begleitung}

Aufgrund der von der Regierung vorgegebenen Sicherheitsvorkehrungen waren einige Caritas-Dienste während den Lockdowns nicht mehr möglich und mussten in ihrer gewohnten Form stark reduziert werden. Dies betraf insbesondere die Beratungsstellen der Caritas. Trotzdem konnten sich einige Dienste anpassen und gingen online. Dies ist der Fall bei der Beratungsstelle zur Existenzsicherung (BEX), die seit April 2020 eine Online-Beratung zu Themen wie Drogenmissbrauch und Sucht, Alkoholmissbrauch, Wohnungsverlust oder akute finanzielle Notlage anbietet. Eine Umstellung und virtuelle Erweiterung waren bereits vor der Pandemie geplant, weil eine Online-Beratung aufgrund der Gewährleistung der vollen Anonymität der KlientInnen und der zeit- und ortsunabhängigen Beantwortung der Fragen von Vorteil ist. In Zeiten von COVID-19 bewahrheitete sich dies umso mehr und die Beratung über Internet und Telefon wurde unerlässlich.

Digitale Medien wurden auch im Bildungskontext eingesetzt. Die acht steirischen Lerncafés bieten SchülerInnen im Alter von 6 bis 15 Jahren ein kostenloses Lern- und Nachmittagsangebot. Die ,LernBars' hingegen bieten kostenlose Lernbetreuung für SchülerInnen von 12 bis 21 Jahren. Während der Pandemie wurden die so genannten ,Lerncafés‘ zu ,Ferncafés'. Als erster Schritt hin zur Online-Begleitung galt es herauszufinden, ob die Kinder und Jugendlichen über die nötigen technischen Möglichkeiten für eine solche Beratungsform verfügen, oder, wie die Leiterin Eva Hödl es sagt, „ob das Kind für uns überhaupt greifbar ist“. Die Initiativen der Stadt Graz oder anderer Organisationen waren nicht in der Lage, alle bedürftigen Familien mit Laptops zu versorgen, sodass die Caritas selbst nach Wegen suchte, diese zu beschaffen. Eva Hödl zufolge waren fast alle Notebook-Bedürfnisse gestillt. Abgesehen von den Kommunikationsmethoden war es das Wichtigste, mit den Kindern in Kontakt zu bleiben:

„Wir hatten Freiwillige, die zusätzlich einmal am Tag oder zwei Mal in der Woche mit dem Kind telefoniert haben, gefragt haben, „Was tut 
sich so?"“, „Wie geht es voran?“..., um diesen Austausch, der im Lerncafé auch enorm wichtig ist, weiter mit den Kindern zu halten. Wir haben einfach viel Zeit gebraucht, um in Kontakt mit den Kindern zu bleiben.“

Dafür mussten sich die PädagogInnen und Freiwilligen an die Bedürfnisse von jedem einzelnen Kind anpassen und das beste Kommunikationsmittel finden. Diese Kontinuität des Kontakts hat ermöglicht, dass kein einziges Kind aus einem Lerncafé während der Pandemie die Schule abgebrochen hat. Die Online-Umstellung war aber nicht die einzige Lösung: So haben PädagogInnen auch Arbeitsunterlagen ausgedruckt und per Post zugeschickt, sofern die betreffende Familie keinen Drucker hatte.

Aufgrund der Lernbedingungen in den Schulen stieg der Bedarf an Unterstützung für sozial benachteilige Kinder während der Pandemiezeit. Die Pandemie hat in der Tat die Ungleichheiten von SchülerInnen im Schulsystem verstärkt. Im Kontext des Fernunterrichts zeigen sich die sozialen Ungleichheiten von Kindern stärker als in ,normalen' Zeiten: fehlende Ressourcen der Eltern (finanziell, zeitlich, sprachlich, Wissen), um ihren Kindern bei den Hausaufgaben zu helfen, fehlender Zugang zu Technologien, oft kein ruhiger Arbeitsplatz und kein von den Eltern definierter und unterstützter Zeitrahmen. Birgit Sauer vom Institut für Politikwissenschaft und Leiterin des Projekts MiCREATE in Wien, spricht in diesem Zusammenhang von einem „Pandemie Gap“, „eine Bildungslücke verursacht durch die Covid-19-Krise“ (Wohlsein 2021). Zusätzlich zu diesen Ungleichheiten beim direkten Zugang zum Fernunterricht haben andere Ungleichheiten während der Krise zugenommen, wie etwa erhöhte Arbeitslosigkeit oder häusliche Gewalt, die Auswirkungen auf die Lernbedingungen von Kindern haben können.

Ein Großteil der SchülerInnen der Fachschule der Caritas in Graz sind sozial benachteilige SchülerInnen. Direktorin Evelyn Awad: „Wir heben viele Schüler aus schwierigen Verhältnissen, viele Migranten, und wir haben eine Abendschule, wo Schüler drinnen sind, die aus dem System gefallen sind und die da wieder einsteigen. Und für diese Klientel war es besonders schwierig." Auch hier war das Wichtigste, den Kontakt mit den SchülerInnen aufrecht zu erhalten: LehrerInnen und die Sozialpädagogin der Schule haben sich viel Zeit genommen, um persönliche Gespräche mit den SchülerInnen zu führen. „Wir haben sie gut betreut, es war viel, viel Arbeit, aber wir haben trotzdem nicht alle erreicht.“, so Evelyn Awad. Ein bis zwei SchülerInnen pro Klasse haben die Schule abgebrochen. Die größte Sorge der Direktorin ist jedoch das allgemeine Wohlbefinden dieser benachteiligten Kinder, denn „Schule ist nicht nur Schule. Schule ist nicht 
nur Lernen. Das ist auch ein Ort der Begegnung, wo man einmal einen Blödsinn macht, Spaß hat und das ist alles weg." Außerdem haben Freizeitaktivitäten (Musik, Sport, Ausflüge) seit einem Jahr nicht mehr stattgefunden, obwohl für diese Gruppe die Schule oft die einzige Möglichkeit bedeutet, diese Art von Aktivitäten auszuüben.

Die Kontinuität des Kontakthaltens konnte nicht überall durch OnlineLösungen aufrechterhalten werden, und vielerorts haben bereits vergessene Methoden wieder ihren Wert gefunden. Einige Pfarrcaritas-Einrichtungden haben beispielsweise fertige Essens- und Hygienepakete durch die Fenster gegeben. Im folgenden Abschnitt wird deutlich, wie jede Einrichtung der Caritas versucht hat, sich anzupassen, um Kontinuität von Nächstenliebe oder dasjenige zu gewährleisten, was Sr. Elisabeth vom Marienstüberl „die Werke der Barmherzigkeit“ nennt. Der Linzer Diözesanbischof Manfred Scheuer hat, in einem Artikel über die Pastoral der Krise in der COVID-19-Pandemie, in diesem Zusammenhang von der „Kreativität der Liebe“ gesprochen. Diese Kreativität der Liebe findet sich in neuen Wegen wieder, die die MitarbeiterInnen der Caritas beschritten haben, um diese Kontinuität der Nächstenliebe zu gewährleisten. Um die Angehörigen zu informieren, wurden neue Formen der Information entwickelt. So etwa wurden in einigen Pflegewohnhäusern Newsletter per Email oder ausgedruckte Informationsbriefe an die Angehörigen verschickt, um den Kontakt aufrecht zu erhalten.

\section{Herausforderungen und neue Nöte erkennen}

Die ungeplante, unstrukturierte und unerwartete Natur von Krisen (Rosenthal 2003) bringt viele Herausforderungen für die MitarbeiterInnen und für die Erfüllung neuer Bedürfnisse, die sich direkt aus der Krisensituation ergeben.

\subsection{Herausforderungen und neue Bedürfnisse für die MitarbeiterInnen}

Die Aufrechterhaltung einer gewissen Normalität oder die Entwicklung einer neuen Normalität erfordern in Zeiten der Krise einen höheren Einsatz der MitarbeiterInnen. In der Krise hat sich gezeigt, dass viele MitarbeiterInnen in der Caritas mit hohem Engagement ihre Aufgaben wahrnehmen, was auch erlaubt hat, in kurzer Zeit auf die neuen Anforderungen, auf neue Not oder auf Problemstellungen zu reagieren. 
Ein Kennzeichen der COVID-19-bedingten Gesundheitskrise ist die lange Dauer und damit verbundene Belastung ohne wirkliche Erholungsphasen. Dies haben besonders die MitarbeiterInnen in den Einrichtungen der Pflege erlebt. Viele haben mit großer Anstrengung und unermüdlichem Einsatz durchgehalten, damit die anstehenden Dienste gut gemacht werden konnten. Wie Christoph Seidl es betont: „Helfende Berufe leisten auch in schwierigen Zeiten das, wozu sie ausgebildet wurden und wozu sie angetreten sind: nämlich anderen in ihrer Not beizustehen, sie zu unterstützen, sie zu entlasten. Aber in einer Pandemie können die eigenen Kräfte rasch an die Grenzen gelangen." (2020 :315) Beide LeiterInnen von Pflegewohnhäusern, mit denen ein Interview geführt wurde, sagen, dass sie in dieser Zeit mehr Gespräche mit MitarbeiterInnen geführt haben. Die mit der Krise verbundenen Arbeitsbedingungen hatten auch Folgen, die über den Arbeitsplatz hinaus in den privaten Bereich hineinreichten. Die LeiterInnen vom Marienstüberl etwa hatten den ganzen Tag Kontakte mit den KlientInnen und waren auf die Möglichkeit einer Infektion vorbereitet. So hatte der Leiter immer einen mit den nötigsten Dingen gepackten Koffer im Büro, für den Fall, dass er sich ansteckte und nach der Arbeit zum Schutz der eigenen Familie nicht mehr zurück nach Hause konnte. Sr. Elisabeth wiederum bewohnte einen anderen Teil ihres Klosters und frühstückte nicht mit den anderen Schwestern. Die PädagogInnen und freiwilligen MitarbeiterInnen der Lerncafés mussten sich von Kindern abgrenzen, die rund um die Uhr Unterstützung brauchten. Um die MitarbeiterInnen zu schützen, wurden die Öffnungszeiten im Lerncafé klar definiert. Auch die LehrerInnen der Fachschule haben hohes Engagement gezeigt, wie die Direktorin es erzählt: „Für die Lehrer war es unglaublich viel Arbeit. Im Normalfall haben sie einen Stundenplan, nun hatten wir praktisch 24 Stunden Dienst und waren immer erreichbar.“

Die Krise hat außerdem eine neue Arbeitsweise gefordert. Es ist grundsätzlich schnell und gut gelungen auf Homeoffice umzustellen und viele Besprechungen online durchzuführen. Die Digitalisierungsschritte waren aber mit neuen Kompetenzen und neuen Geräten verbunden, an die die MitarbeiterInnen sich schnell anpassen mussten. Die Leiterin der Lerncafés berichtet dahingehend: „Es hat fast jeder im ersten Lockdown ein Handy bekommen. Meine Mitarbeiter sind nicht die höchste Einkommensschicht und sie haben kein Smartphone oder ein Wertkartenhandy. [...] Also, wir mussten einige Geräte austeilen, WhatsApp freischalten lassen von der IT.“ Die PädagogInnen von den Lerncafés mussten selber lernen, ein Smartphone und ,Zoom ' zu verwenden. Digitalisierungsworkshops über Lernmethoden im Online-Bereich wurden für sie organisiert. 
Diese Krise hat letztlich auch gezeigt, dass Spannungen, die in der Gesellschaft sind, auch bei MitarbeiterInnen spürbar sind. Neben jenen, die sehr aktionsorientiert in der Krise arbeiten und neue Projekte entwickeln, gibt es auch jene, die eher sicherheitsorientiert, vielleicht auch ängstlich, alle Regeln möglichst genau umsetzen wollen, vielleicht sogar zusätzliche Sicherheitsmaßnahmen vorschlagen. Besonders in dieser Krise hat sich gezeigt, dass die Fähigkeit, mit Unsicherheiten umzugehen, sehr unterschiedlich ausgeprägt ist, vielleicht auch deswegen, weil die Caritas in den letzten Jahren viel investiert hat in die Entwicklung von Strategien und langfristigen Planungen. Plötzlich war es notwendig, auf Sicht zu agieren und sehr kurzfristig zu entscheiden. Das hat manche sehr gefordert und Führungskräfte mussten mit diesen unterschiedlichen Erwartungen gut umgehen. Eine wesentliche Aufgabe war es plötzlich, Spannungen auszuhalten und trotzdem Sicherheit und Klarheit zu vermitteln.

\subsection{Neue Bedürfnisse bei den Obdachlosen}

Was heißt eine Quarantäne oder Ausgangsperre für diejenigen, die kein Dach über dem Kopf haben? Wie kann man ,daheim bleiben', wie es beim ersten Lockdown das Motto war, wenn man kein Zuhause hat? Wohnungslose Menschen wurden also während der Pandemie mit neuen Herausforderungen konfrontiert, auf die die Caritas zu antworten versucht hat. Eine erste Antwort darauf war, dass die Notschlafstellen während des Lockdowns auch tagsüber geöffnet hatten - im Gegensatz zu den herkömmlichen Öffnungszeiten vom Abend bis in der Früh. Eine Frage, die bei der Caritas in der ersten Woche des Lockdowns schnell aufkam, war, was zu tun ist, wenn sich eine Person mit möglichen COVID-19-Symptomen in einer Notschlafstelle vorstellt. Es ist nicht möglich, Menschen am Abend nicht aufzunehmen, ohne ihnen einen alternativen Schlafplatz anzubieten. Um diesem potenziellen Bedarf gerecht zu werden, hat die Caritas ein Quarantäne-Wohnungssystem für Verdachtsfälle eingerichtet. MitarbeiterInnen von anderen Einrichtungen und Freiwillige hatten die Aufgabe, die Menschen von der Notschlafstelle in die Wohnung zu bringen und sie während der zweiwöchigen Quarantäne jeden Tag zu besuchen. Nach und nach erhielt die Caritas Unterstützung von der Stadt Graz mit weiteren Wohnungen, die genutzt werden konnten, und Ende April 2020 stellte ein Hotel der Caritas sogar Zimmer zur Verfügung und machte damit gesellschaftliche Solidarität erfahrbar.

Wie schon erwähnt, wurden die Angebote in der Suppenküche des Marienstüberls an die individuelle Situation angepasst. Obdachlose Menschen 
und diejenigen, die nicht zu Hause essen konnten, durften weiterhin im Speisesaal essen. So wurde für diejenigen, die weniger in den normalen Mustern der Gesellschaft leben, größtmögliche Normalität bewahrt, um eine Form von Sicherheit zu erhalten. Wie Anne-Françoise Dequiré in einem Artikel über Obdachlose schrieb: „Innerhalb der Norm zu bleiben, scheint wichtig zu sein und erlaubt es dem Einzelnen, sich selbst zu beruhigen" (Dequiré 2010). Weil ein großer Teil von Normalität wegfällt, ist es umso wichtiger, diese in Krisenzeiten auf der Makro-Ebene zu bewahren.

\subsection{Zunehmende Einsamkeit}

Einsamkeit ist eine wachsende Plage in unserer Gesellschaft. Einpersonenhaushalte sind bei jungen und älteren Menschen im Steigen begriffen und können zu Formen von Einsamkeit führen, die die Pandemie mit ihren sukzessiven Lockdowns und sogenannten ,Social Distancing' ernsthaft verschlimmert hat. 2019 waren laut Statistik Austria 37,5 Prozent der Haushalte in Österreich Einpersonenhaushalte - großteils mit Menschen über 65 Jahre. Was kann die Caritas, deren Motto ,wir $>$ ich ' (Wir ist größer als ich) lautet, leisten? Einsamkeit betrifft vor allem Menschen, die nicht in die üblichen Netzwerke einer Gesellschaft, wie Familie oder Arbeitsplatz, eingebunden sind. Obdachlose Menschen sind besonders von Einsamkeit betroffen. Sr. Elisabeth betont: „Die Einsamkeit hat viele schon sehr gequält, das haben sie auch gesagt." Das Marienstüberl ist nicht nur als Essensausgabe gedacht, sondern auch als Begegnungsstätte für Menschen, die am Rande der Gesellschaft leben. Diese Menschen besuchten sich früher gegenseitig in Heimen, in denen sie sich aufhielten, was bald pandemiebedingt nicht mehr möglich war. Während des Gesprächs mit Sr. Elisabeth ruft eine Dame, eine Klientin des Marienstüberls, die sich derzeit in einem Heim befindet, mehrmals am Telefon der Schwester an. Sr. Elisabeth erklärt, dass sie mehrmals am Tag anruft, weil sie derzeit sehr einsam ist, ohne Besuch. Sr. Elisabeth hebt ab, um mit der Frau das Abendgebet zu sprechen und ihr eine gute Nacht zu wünschen. Sie rezitiert ein Gebet zu den Schutzengeln und ein Vaterunser und segnet sie, indem sie das Kreuzzeichen auf das Telefon nachzeichnet. Laut Sr. Elisabeth wären genau das der Auftrag und die Identität der Caritas, nämlich in Notzeiten da zu sein für Menschen und mit den gewohnten Aufgaben weiterzumachen:

„Wir können die Armen nicht auf die Straße sperren. Wir können sie nicht verhungern lassen. Das ist unser Auftrag und das ist das 
wichtigste, was immer kommt, es muss dieser Auftrag bleiben, dass man den Menschen zu essen und zu trinken gibt und Wärme. Das bleibt. Das sind die Werke der Barmherzigkeit, die muss man tun, ob es jetzt erlaubt ist oder nicht erlaubt, das gebietet die Liebe und das ist eigentlich Caritas, gelebte Caritas. Wenn wir das nicht mehr tun, sind wir nicht Caritas.“

Doch die Einsamkeit während der Pandemie betraf nicht nur Menschen, die bereits am Rande der Gesellschaft standen. Das ,Team Nächstenliebe', ein Telefon- und Online-Netzwerk der Caritas Österreich, das bereits zu Beginn des ersten Lockdowns eingerichtet wurde, um Bedürfnisse und Hilfsangebote zu ermitteln, zeigt eine andere Form von Bedürftigkeit. Das Profil der Hilfsbedürftigen war laut Katharina Fink, zuständig für das Team Nächstenliebe in Graz, eine „breite Palette“. Am Anfang meldeten sich hauptsächlich ältere Leute, die meist um Hilfe fürs Einkaufengehen anfragten. Katharina Fink betont:

„Was mir nicht bewusst war, ist, dass es einfach extrem viele Menschen gibt, die kein Netz haben, wirklich, oder keine vertrauten Personen, die sie fragen können, wenn es auch nur eine Kleinigkeit ist. Man fühlt sich nicht fit und braucht etwas vom Supermarkt. Ich habe am Anfang oft naiv gefragt, ob sie nicht einen Freund, oder einen Nachbarn oder ein Kind haben, sie haben gesagt: nein, sie wüssten nicht, wen sie fragen sollten."

In diesem Sinne hat die Krise andere, stillere Nöte unserer Gesellschaft geoffenbart, die bislang nicht gesehen wurden. In letzter Zeit haben sich Menschen beim „Team Nächstenliebe“ gemeldet, weil sie jemanden suchten, mit dem sie spazieren gehen und Kontakt haben können - „weil es zu zweit netter ist“, wie Katharina Fink berichtet. „Jetzt, nach einem Jahr, geht es sehr stark in Richtung Einsamkeit.“

\subsection{Die neue Armut}

Kurzarbeit, Home-Schooling, Jobverlust: Die viel zitierte ,Corona-Krise hat die Situation jener verschlimmert, die schon vorab von Armut bedroht waren und darüber hinaus Menschen in Notlagen gebracht, die zuvor noch nie von Armut bedroht waren. Die österreichische Armutskonferenz berichtet:

„Wer vor dem März 2020 prekär oder gar irregulär gearbeitet hatte, konnte in den Wochen danach seinen bzw. ihren Lebensunterhalt aus 
eigener Kraft kaum noch bestreiten. Wer in prekären Verhältnissen arbeitet, kann einen wochenlangen Lockdown bzw. eine noch längere Zeit mit geringerem oder gar keinem Erwerbseinkommen nicht überbrücken.“ (Österreichische Armutskonferenz 2020)

Die Auswirkungen dieser ,neuen' Armut werden erst nach und nach in den verschiedenen Caritas-Organisationen zu spüren sein. Im Marienstüberl beispielsweise gab es im vergangenen Jahr eigentlich weniger KlientInnen - nicht zuletzt, weil weniger Menschen im Zuge von armutsbedingter Arbeitsmigration aus dem Ausland kamen und Unterstützung brauchten. Sr. Elisabeth zufolge sind auch Menschen ferngeblieben aufgrund von Angst. Außerdem stellt die Suppenküche für viele eine Art Hemmschwelle dar: Viele Bedürftige stellen sich erst dann um ein warmes Essen an, nachdem alle anderen Formen von Hilfe ausgeschöpft worden sind. Innerhalb des Caritas-Netzwerks ist die erste Anlaufstelle für Menschen, die von dieser neuen Armut betroffen sind, die Pfarrgemeinde. In den ländlichen Gebieten und Regionen außerhalb von Graz war diese Form der Armut schon bei der Lebensmittelausgabe spürbar.

„Es sind sehr viele Familien dazu gekommen bei der Lebensmittelausgabe, die wirklich arbeitslos sind, oder Alleinerziehende, die die Nebenjobs nicht mehr ausüben können und uns sagen, dass sie seit Corona nicht mehr ,über die Runden' kommen. Man merkt: von Monat zu Monat werden es mehr.“ (Gabriele Anderssohn)

Laut Caritas-Regionalkoordinatorin Anderssohn ist die Zahl Bedürftiger etwa in Schladming stark angewachsen. Die Krise hat diejenigen, die ohnehin schon gefährdet waren, besonders hart getroffen und Menschen weiter in die Armut getrieben. Um diesen neuen Bedarf zu decken, hat die Caritas einen Spendenaufruf gestartet, für Menschen im Inland, die sich Heizung, Essen oder Wohnung nicht mehr leisten können.

\section{Systemrelevanz in der Krise: Kurz-und Langfristigkeit}

\subsection{Caritas intern}

Die Stärke der Caritas in einer Krise liegt in ihrer Fähigkeit, sich schnell an neue und unvorhergesehene Situationen anzupassen. Die interviewten Führungskräfte und MitarbeiterInnen betonen, wie ihre Einrichtung sich trotz Unklarheit der Maßnahmen schnell den Gegebenheiten anpassen konnte. Eva Hödl etwa berichtet von den Lerncafés, dass es am 16. März, 
dem ersten Tag ohne Lernbetrieb, jedem Kind klar war, wer seine PädagogIn ist. Auch im Marienstüberl erklärt Philipp Friesenbichler: „Da bin ich dankbar, dass es [ein geregelter Ablauf] relativ rasch gekommen ist. Wir haben relativ rasch zusätzliche Zivildiener gekriegt.“ Auch in Bezug auf die Impfung Anfang 2021 hat die Caritas schnell reagiert. Klaus Sauseng, der auch im Krisenstab der Caritas ist, erklärt: „Wir, die Caritas, waren die ersten in der Steiermark, die geimpft worden sind. Zwei Tage, bevor die anderen geimpft haben. Der offizielle Impfstart war zwei Tage später. [...] Die Caritas war in der Umsetzung sehr bemüht, sehr schnell, sehr zielstrebig."

Diese schnelle Anpassung innerhalb der Caritas-Einrichtungen wurde in erster Linie durch die Vielzahl unterschiedlicher, interner Ressourcen ermöglicht. Schon im Mai 2020 betonte Caritas-Direktor Herbert Beiglböck: „Hier zeigt sich der Vorteil der Größe der Caritas, dass in diesem Fall relativ leicht interne Umschichtungen möglich sind, weil die Aufgabenstellungen sehr ähnlich sind.“ (Beiglböck 2020). So wurden MitarbeiterInnen anderer Einrichtungen in die Pflegewohnhäuser entsandt, um auszuhelfen. Die Leiterin des Pflegewohnhauses in Hitzendorf berichtet:

„Das war wirklich sehr schön finde ich, wir haben da drei Kolleginnen gehabt, die aus der Familienhilfe gekommen sind, und das war eine große Bereicherung. Da war mehr individuelle Betreuung möglich. Das war schon faszinierend in dieser Zeit. Wenn beispielsweise Corona in einem Haus ausgebrochen ist, und man hat einen Aufruf gemacht und gefragt, wer wäre bereit auch dort zu helfen - da war die Bereitschaft sehr hoch. Das ist auch schön zu beobachten, denke ich mir.“

Die Arbeit der Caritas stützt sich nicht nur auf bezahlte MitarbeiterInnen, sondern auch auf Ehrenamtliche - im Jahr 2019 waren es insgesamt 1.601 Menschen. Das Profil der Freiwilligen weist ein hohes Durchschnittsalter auf, was bedeutet, dass diese Arbeitskräfte während der Pandemie als Risikogruppe angesehen wurden und in den Einrichtungen nicht mehr weiterhelfen konnten. Dank ihrer Netzwerke war die Caritas jedoch in der Lage, schnell neue Freiwillige zu finden.

\subsection{Dauerhafte Lösungen?}

Krise meint immer eine ,Ausnahmezeit', außerhalb der Normalität. Einige der von der Caritas während dieser Krise angebotenen Lösungen werden mit großer Wahrscheinlichkeit Bestand haben - zum einen, weil die so- 
zialen Auswirkungen der Krise zweifellos in den nächsten zwei bis drei Jahren zu spüren sein werden, und zum anderen, weil einige der Angebote tatsächlich auf Bedürfnisse reagiert haben, die unabhängig von der viel zitierten ,Corona-Krise' vorhanden waren. Als Beispiel ist hier das ,Team Nächstenliebe ${ }^{6}$ zu nennen, das nicht nur auf die Bedürfnisse derjenigen reagiert, die einsam sind, sondern auch auf den Wunsch von Freiwilligen, die sich nur für eine kurze Zeit oder für eine bestimmte Aktion engagieren wollen. „Es gibt Freiwillige, die sich genau deshalb melden, weil sie etwas gegen die Einsamkeit tun wollen. ,Gegen die Einsamkeit' ist eine neue Kategorie“ (Katharina Fink). Die neue Kategorie erfordert neue Formen von Unterstützung.

Andere Angebote, wie etwa die Lebensmittelausgabe in den Regionen, sind auch über die Zeit der Krise hinaus nützlich: „Die Lebensmittelausgabe war eigentlich als Übergangslösung gedacht, bis die anderen Institutionen wieder öffnen, aber das ist eine fixe Einrichtung bei uns geworden, ohne die es nicht mehr gehen würde. Es wäre ganz schlimm für viele Leute, denke ich, wenn wir damit aufhören würden" (Gabriele Anderssohn). Die Lebensmittelausgabe reagiert zum Teil auf einen Bedarf, der zweifellos bereits vor COVID-19 vorhanden war, aber auch auf die gestiegene Anzahl bedürftiger Familien aufgrund der sozialen Folgen der Pandemie. Auch die Lerncafés werden sich nachhaltig mit den Folgen der Krise auseinandersetzen müssen - insbesondere, um den in den Jahren 2020 und 2021 entstandenen Lernmangel auszugleichen. Nach Aussage der Leiterin wird es in den nächsten zwei Jahren notwendig sein, die Begleitung der Kinder und Jugendlichen zu intensivieren. Gleichzeitig hat die Krise Veränderungen mit sich gebracht, insbesondere im Bereich der Digitalisierung. Während es vorher nur einen Computer in den Lerncafés für die PädagogInnen gab, bleiben einige Tablets und Laptops, die während der Krise angeschafft, wurden nun dort und werden bis heute als Lehrmittel eingesetzt. Was die Digitalisierung der Arbeit anbelangt, so wird einiges davon auch in Zukunft beibehalten werden und die Arbeitsgestaltung der Caritas-MitarbeiterInnen dauerhaft verändern. Dies zeigt sich an der bereits beschlossenen ,Betriebsvereinbarung Homeoffice' und einer damit verbundenen, angedachten Reduktion von Büroflächen. Eine solche Entwicklung kann Chance sein, birgt aber auch Gefahren hinsichtlich ungleicher Lebens- und Arbeitsbedingungen der einzelnen MitarbeiterInnen. Außerdem bringt eine solche Entwicklung Herausforderungen mit sich, betreffend Teamentwicklung und dem gemeinsamen Gestalten und Entwickeln von strategischen Inhalten und Projekten. Auch die Frage nach der bestmöglichen Integration von MitarbeiterInnen mit weniger ausgeprägten digitalen Fähigkeiten (und im Gegenzug dafür oft hoher 
sozialer Kompetenz) stellt sich. Gibt es auch für sie in Zukunft gute Orte des Arbeitens, wo sie sich mit ihren Fähigkeiten und ihrer Zuwendung zum Menschen einbringen können? All das erfordert eine gute intensive Reflexionsphase, damit zukünftige Herausforderungen zur Zufriedenheit der Beteiligten gelöst werden können und es zu keinem Nebeneinander von alten Mustern und neuen Modellen kommt, die unreflektiert übernommen und weitergeführt werden.

\subsection{Spirituelle Ressourcen der Caritas}

Studien über Religion als Bewältigungsmechanismen in schwierigen Situationen - oder sogenannte Copingstrategien - zeigen aus objektiver Sicht, wie spirituelle Ressourcen den einzelnen Menschen befähigen, persönliche oder soziale Krisen besser zu bewältigen (Pargament/Park 1997). Interviews mit den Führungskräften der verschiedenen Caritas-Einrichtungen zeigen, dass Spiritualität als Ressource in Krisenzeiten eine wichtige Rolle spielt. An dieser Stelle sei die Reaktion von Caritas-Angestellten auf die seitens der Direktion an die Mitarbeitenden ausgeschickten Emails angeführt. In diesen ein- bis zweimal im Monat versendeten Emails ergeht an die Caritas-Bediensteten neben arbeitsrelevanten Informationen auch jeweils ein geistlicher Impuls. Gerade dieser erwies sich während der COVID-19-Pandemie für so manche MitarbeiterInnen als richtungsweisend und tröstlich. "Danke für Ihre tröstenden und aufmunternden Worte. Ich finde auch Unterstützung im Psalm 91", antwortet eine Empfängerin dem Caritas-Direktor auf eine seiner Aussendungen per Email. Ein ganz konkretes Beispiel für gelebte Spiritualität ist in der Caritas-Einrichtung Marienstüberl zu finden. So beten beispielsweise die beiden Leitungspersonen des Marienstüberls seit letztem März nach der Arbeit gemeinsam - um, wie sie selbst sagen, die nötige Energie für die weitere Arbeit zu schöpfen. Sr. Elisabeth erzählt: „Wir haben auch unser Corona-Gebet gemacht. Nur wir zwei, ab und zu, wenn jemand da ist, beten wir gemeinsam. Das ist letztlich der Glaube, der trägt, und die Caritas ist auch ein Glaubensträger. [...] Dienst und Wort, du kannst das nicht trennen." Der Leiter der Pflegewohnhäuser in Rottenmann und Frauenberg bestätigt das ebenfalls sowohl seitens der MitarbeiterInnen als auch seitens der BewohnerInnen:

„Wenn ein Bewohner, eine Bewohnerin, gestorben ist, haben wir auch im Andachtsraum ein Bild angebracht, wir haben einen Seelsorger gehabt, der ins Haus gekommen ist. Diese spirituelle Haltung war gut, 
und sie war wichtig und im richtigen Zeitpunkt richtig, gut, richtig und wichtig. Auch für die Mitarbeiter - definitiv.“ (Klaus Sauseng)

Für die KlientInnen vom Marienstüberl bildet auch der Glaube eine wichtige Stütze in schwierigen Zeiten. Sr. Elisabeth, die mit den Gästen in der Suppenküche täglich betet, erzählt:

„[I]ch habe bemerkt, das war für sie [die BesucherInnen des Marienstüberls] der Halt. Weil letztlich: Wo wenden wir uns hin? Was haben wir noch? Wir haben nichts! Und das habe ich beibehalten. [...] Dieses Getragensein durch das Gebet - das ist es, was wir als Christen haben, dass wir sagen, Gott ist stärker, es gibt überall einen Sinn drinnen, und wir müssen darauf kommen. Aber Gott meint es immer gut mit uns. Sogar in der Corona Zeit, wir haben nie aufgehört damit, das zu glauben. Wir wenden uns an Gott - wohin sonst sollten wir uns wenden? Wir haben immer Hoffnung, das ist unser Glaube.“ (Sr. Elisabeth)

Eine der Stärken in der Caritas ist die Fähigkeit, in Krisenzeiten schwierige Situationen zu überwinden, in Rückbesinnung auf die Religion. Beispiele wie das angeführte verdeutlichen, dass solche spirituellen Ressourcen nicht nur Teil der Identität der Caritas als Organisation sind, sondern auch von den MitarbeiterInnen gelebt und genutzt werden.

\section{Conclusio: Die Krise als Ort der Hoffnung und Dankbarkeit}

Aus christlicher Sicht ist die Krise auch ein Ort der Hoffnung und der Dankbarkeit. Im April 2020 machte Caritas Direktor Herbert Beiglböck gemeinsam mit Andrea Ederer, Präsidentin der Katholische Aktion Steiermark, und dem Integrationsbeauftragten der Diözese Graz-Seckau, Erich Hohl, in einer Aussendung deutlich: "Wir halten es für angebracht, jetzt ein Zeichen solcher Dankbarkeit zu setzen, indem wir Flüchtlinge in Österreich aufnehmen. [...] Wie wäre es zum Beispiel, wenn wir für jeweils hundert Genesene einen gefährdeten Menschen aus einem Lager in Griechenland aufnehmen?" (Katholisch 2021)

Die Krise hat auch Formen neuer Solidarität zu Tage gebracht. So konnte bei den Geldspenden ein Plus gegenüber dem Zeitraum vor der Corona-Krise von 15 Prozent verzeichnet werden und auch die Anzahl der Freiwilligen, sowohl in den Einrichtungen als auch in den Pfarren, hat sich signifikant erhöht. Wenn während des Schutzmaterial-Mangels Frauen aus der Dorfgemeinschaft in Hitzendorf ehrenamtlich, unentgeltlich und in Eigeninitiative Mund-Nasenschutz-Masken für die Mitarbeite- 
rinnen des Pflegewohnhauses genäht haben, ist das nur ein Beispiel von gelebter Solidarität, die im Zuge der Krise entstanden ist. Solidarisches Handeln gibt Anlass zur Hoffnung auf neue Formen des Zusammenhaltes innerhalb der Gesellschaft. Ein Weniger an Sozialleben, wie es in den pandemiebedingten Lockdowns der Fall war, muss nicht automatisch ein Weniger an Solidarität bedeuten. Die viel zitierte Krise wird so zum Ort der Hoffnung und der Dankbarkeit für kontinuierlich erfahrene Nächstenliebe.

\section{Literatur}

Alonso, Juan/Bertrand, Denis: Du corps normalisé aux normalités sociales, in: Dies. : Formes et figures de la normalité (2011), in: https://f.hypotheses.org/w p-content/blogs.dir/2011/files/2014/07/Figures-et-formes-de-la-normalit\%C3\% A9-Dossier-de-recherche-en-s\%C3\%A9miotique-de-lEcole-de-la-Communicatio n-de-SciencesPo-Paris-sous-la-direction-de-Juan-Alonso-et-Denis-Bertrand-1.pdf [27.04.2021].

Beiglböck, Herbert: Caritas in Zeiten von Corona, in: Feinschwarz (01.05.2020), in: https:/www.feinschwarz.net/caritas-in-zeiten-von-corona/ [26.05.2021].

Caritas: Homepage der Caritas Steiermark, in: https://www.caritas-steiermark.at/ [26.05.2021].

Dequiré, Anne-Françoise: Le Corps des sans domicile fixe, in: Recherches \& éducations, 2010, in: http://journals.openedition.org/rechercheseducations/572 [04.05.2021].

Katholisch, in https://www.katholisch.at/aktuelles/129801/dioezese-graz-seckau-aus -dankbarkeit-fluechtlinge-aufnehmen [21.06.2021].

Österreichische Armutskonferenz: „Armutsbetroffene und die Corona-Krise. Eine Erhebung zur sozialen Lage aus der Sicht von Betroffenen“ (2020), in: https://w ww.armutskonferenz.at/files/armutskonferenz_erhebung_armutsbetroffene_cor ona-krise_2020.pdf [09.06.2021].

Pargament, Kenneth I./Park, Crystal L.: In times of stress: The Religion-Coping Connection, in: B. Spilka/ McIntosh D.N. (Hg.): The Psychology of Religion: Theoretical approaches. Westview Press 1997, 43-53.

Rosenthal, Uriel: September 11: Public Administration and the Study of Crises and Crisis Management, in: Administrative Society 35/2 (2003) 129-143.

Roux-Dufort, Christophe: Gérer et décider en situation de crise: Outils de diagnostic de prévention et de décision. Paris: Dunod 2003.

Scheuer, Manfred: Die COVID-19-Pandemie aus der Sicht eines Diözesanbischofs (20.11.2020), in: https://www.dioezese-linz.at/dl/LkMtJLJLnkNJqx4KJK/2020_1 1_20_Vortrag_Fachtagung_Liturgie_und_Covid-19_KU_Linz_pdf [04.05.2021]. 
Seidl, Christoph: „Sein Unglück ausatmen können“. Hilfe für die Helfenden, in: Kröll, Platzer, Ruckenbauer, Schaupp, Die Corona-Pandemie. Ethische, gesellschaftliche und theologische. Reflexionen einer Krise, Baden-Baden: Nomos (2020) 313-328.

Wohlsein, Barbara: Der „Pandemie Gap“ in der Bildung (17.11.2020), in: https://m edienportal.univie.ac.at/uniview/wissenschaft-gesellschaft/detailansicht/artikel/d er-pandemie-gap-in-der-bildung/?no_cache=1 [10.06.2021].

\section{Erfahrungsberichte}

Gabriele Anderssohn (Regionalkoordinatorin Ennstal \& Ausseerland)

Evelyn Awad (Direktorin der Fachschule für wirtschaftliche Berufe in Graz)

Katharina Fink (Regionalkoordinatorin Graz, Team Nächstenliebe)

Anita Freismuth-Jauschneg (Hausleiterin Pflegewohnhaus in Hitzendorf)

Philipp Friesenbichler (Marienstüberl, Graz)

Sr. Elisabeth Gruber (Marienstüberl, Graz)

Eva Hödl (Leitung Lerncafés \& Lernbars)

Klaus Sauseng (Hausleiter Pflegewohnhäuser in Rottenmann und Frauenberg, Mitglied des Krisenstabs der Caritas Steiermark) 
